ARTICLE

DOI: $10.1038 / s 41467-018-04326-1$

\title{
Emergent superconductivity in an iron-based honeycomb lattice initiated by pressure-driven spin-crossover
}

Yonggang Wang (1) ${ }^{1,2}$, Jianjun Ying (1) ${ }^{3,4}$, Zhengyang Zhou ${ }^{5,6}$, Junliang Sun ${ }^{5}$, Ting Wen ${ }^{1}$, Yannan Zhou ${ }^{7}$, Nana Li ${ }^{1}$, Qian Zhang ${ }^{1}$, Fei Han ${ }^{1,2}$, Yuming Xiao ${ }^{4}$, Paul Chow ${ }^{4}$, Wenge Yang ${ }^{1,2}$, Viktor V. Struzhkin ${ }^{3}$, Yusheng $\mathrm{Zhao}^{8}$ \& Ho-kwang Mao ${ }^{1,3}$

The discovery of iron-based superconductors (FeSCs), with the highest transition temperature $\left(T_{c}\right)$ up to $55 \mathrm{~K}$, has attracted worldwide research efforts over the past ten years. So far, all these FeSCs structurally adopt FeSe-type layers with a square iron lattice and superconductivity can be generated by either chemical doping or external pressure. Herein, we report the observation of superconductivity in an iron-based honeycomb lattice via pressuredriven spin-crossover. Under compression, the layered $\mathrm{FePX}_{3}(X=\mathrm{S}, \mathrm{Se})$ simultaneously undergo large in-plane lattice collapses, abrupt spin-crossovers, and insulator-metal transitions. Superconductivity emerges in $\mathrm{FePSe}_{3}$ along with the structural transition and vanishing of magnetic moment with a starting $T_{\mathrm{c}} \sim 2.5 \mathrm{~K}$ at $9.0 \mathrm{GPa}$ and the maximum $T_{\mathrm{c}} \sim 5.5 \mathrm{~K}$ around $30 \mathrm{GPa}$. The discovery of superconductivity in iron-based honeycomb lattice provides a demonstration for the pursuit of transition-metal-based superconductors via pressure-driven spin-crossover.

\footnotetext{
${ }^{1}$ Center for High Pressure Science and Technology Advanced Research (HPSTAR), 100094 Beijing, China. ${ }^{2}$ HPSynC, Geophysical Laboratory, Carnegie Institution of Washington, Argonne, IL 60439, USA. ${ }^{3}$ Geophysical Laboratory, Carnegie Institution of Washington, Washington, DC 20015, USA. ${ }^{4}$ HPCAT, Geophysical Laboratory, Carnegie Institution of Washington, Argonne, IL 60439, USA. ${ }^{5}$ College of Chemistry and Molecular Engineering, Peking University, 100871 Beijing, China. ${ }^{6}$ College of Chemistry and Chemical Engineering, Chongqing University, 400044 Chongqing, China. ${ }^{7}$ Institute of Nanostructured Functional Materials, Huanghe Science and Technology College, 450006 Zhengzhou, China. ${ }^{8}$ Southern University of Science and Technology, 518055 Shenzhen, China. These authors contributed equally: Yonggang Wang, Jianjun Ying, Zhengyang Zhou, Junliang Sun. Correspondence and requests for materials should be addressed to W.Y. (email: yangwg@hpstar.ac.cn) or to V.V.S. (email: vstruzhkin@carnegiescience.edu) or to Y.Z. (email: zhaoys@sustc.edu.cn)
} 
S nce the discovery of $26 \mathrm{~K}$ superconductivity (SC) in $\mathrm{LaO}_{1}$ ${ }_{x} \mathrm{~F}_{x} \mathrm{FeAs}$ in $2008^{1}$, the Fe-based superconductors (FeSCs) have attracted enormous research interest, owing to their rich compositional and structural varieties ${ }^{1-12}$. The rapid development on this new SC family has led to the highest critical temperature $\left(T_{\mathrm{c}}\right)$ up to $55 \mathrm{~K}$ in $\mathrm{F}$-doped $\mathrm{SmFeAsO}^{2}$ and the discovery of a large number of FeSCs with various structure types ${ }^{3,5-12}$. Similar to the high- $T_{\mathrm{c}}$ cuprate family unexceptionally adopting square $\mathrm{CuO}_{2}$ layers, all of these FeSCs structurally possess infinite antifluorite-like $\mathrm{Fe}_{2} X_{2}$ layers comprising the stacks of edge-sharing $\mathrm{Fe}_{4}$ tetrahedra ( $X$ denotes a pnictide or a chalcogenide element). Therefore, it is widely believed that the common $\mathrm{Fe}_{2} X_{2}$ layer is the essential building unit for the rational structure design of FeSCs, similar in nature to the $\mathrm{CuO}_{2}$ unit in cuprate SCs. And this viewpoint is verified by the discovery of high- $T_{\mathrm{c}} \mathrm{SC}$ in a large number of FeSe-derived layered compounds such as $A_{x} \mathrm{Fe}_{2-y} \mathrm{Se}_{2}(A=\text { alkali metals })^{9,10}, \mathrm{Li}_{x}\left(\mathrm{NH}_{2}\right)_{y}\left(\mathrm{NH}_{3}\right)_{1}$ ${ }_{-y} \mathrm{Fe}_{2} \mathrm{Se}_{2}{ }^{11}$, and $\left(\mathrm{Li}_{0.8} \mathrm{Fe}_{0.2}\right) \mathrm{OHFeSe}{ }^{12}$. Another key feature of FeSCs is a well-recognized fact that in most cases the high- $T_{\mathrm{c}} \mathrm{SC}$ can emerge by suppressing the long-range antiferromagnetic (AFM) order in the stoichiometric parent compounds. Experimentally, two general routes are adopted to achieve this purpose: first, chemical doping or substitution, such as $\mathrm{F}$ doping in $\mathrm{REFeAsO}^{1}$ (RE = rare earth elements) and interlayer cation doping (alkali metals or $\left.\mathrm{NH}_{3}\right)^{9-11}$; second, application of chemical or external pressure, such as the replacement of $X$ in $\mathrm{Fe}_{2} X_{2}$ by congeners with bigger or smaller atomic radii or the substitution of smaller RE ions for La ions ${ }^{2}$.

Under applied high pressure (HP), materials undergo direct structural evolutions including the shortening of metal-ligand bond length, the distortions in the nearest neighbor environment, and the introduction of stress. These structural evolutions make HP significant in the researches on SCs, and many unanticipated SCs have been discovered with this powerful tool. A recent famous example is the observation of conventional SC with high$T_{\mathrm{c}}$ in hydrogen-rich systems, $\mathrm{H}_{x} \mathrm{~S}$, and $\mathrm{PH}_{3}{ }^{13,14}$, under HPs up to megabar scale. Besides, unexpected SC phase diagrams (e.g., $\mathrm{MnP}^{15}, \mathrm{Tl}_{0.6} \mathrm{Rb}_{0.4} \mathrm{Fe}_{1.67} \mathrm{Se}_{2}$, and $\mathrm{K}_{0.8} \mathrm{Fe}_{1.7+\delta} \mathrm{Se}_{2}{ }^{16}$ ) and greatly enhanced $T_{\mathrm{c}}$ values in both cuprates and FeSCs (e.g., $\mathrm{LaO}_{1}$ ${ }_{-x} \mathrm{~F}_{x} \mathrm{FeAs}^{17}, \mathrm{HgBa}_{2} \mathrm{Ca}_{2} \mathrm{Cu}_{3} \mathrm{O}_{8+\delta}{ }_{18}$, and $\beta-\mathrm{Fe}_{1.01} \mathrm{Se}^{19}$ ) have also been successfully achieved by pressure tuning. Moreover, applying external pressure to transition metal (TM) systems may also lead to other significant phenomena such as large-volume collapse $\mathrm{e}^{20-22}$, spin-crossover $(\mathrm{SCO})^{22-24}$, charge disproportionation $^{25}$, and insulator-metal transition (IMT). Regarding the pressure-driven SCO, in which magnetic ions undergo high-tolow spin-state transition, it is possible to produce a nonmagnetic phase through the pressure-induced spin-quenching $(S=0)$. Keeping the relationship between magnetism and SC in mind, an adventurous but meaningful idea is: can we make new nonmagnetic FeSCs without the FeSe-type structure via pressuredriven spin-quenching? If so, $\mathrm{Fe}^{2+}$ with $d^{6}$ electrical configuration in a sixfold-coordinated environment should be an ideal candidate to ensure a nonmagnetic HP phase with zero spin ground state $(S=2$ to $S=0)$.

In the pursuit of abrupt pressure-driven SCOs in TM chalcogenides, we became aware of the essential role of the TMsublattice dimensions in the cooperativeness of $\mathrm{SCO}^{26,27}$. We have discovered abrupt pressure-driven phase transitions accompanied by a high-to-low spin-state transition of $\mathrm{Mn}^{2+}$ in the transition from three-dimensional (3D) NaCl-type $\mathrm{MnX}$ to two-dimensional (2D) $\mathrm{MnPX}_{3}(X=\mathrm{S}, \mathrm{Se})$ with an $\mathrm{Mn}^{2+}$ honeycomb lattice. Subsequent experiments showed that similar cooperative SCO phenomenon also exists in the iron homologs $\mathrm{FePX}_{3}$, in which $\mathrm{Fe}^{2+}$ ions $\left(d^{6}\right)$ arranged on a nearly perfect honeycomb lattice undergo a sharp magnetic-to-nonmagnetic transition $(S=2$ to $S=0)$. This $2 \mathrm{D}$ system apparently meets all the above-mentioned prerequisites for the emergence of SC. In this letter, we report our discoveries in the course of HP studies of the layered compound $\mathrm{FePX}_{3}(X=\mathrm{S}$, Se). Along with the pressure-driven SCO, in-plane lattice collapse, and semiconductor-to-metal transition, SC emerges around $3 \sim 5 \mathrm{~K}$ in the nonmagnetic $\mathrm{HP}$ phase of $\mathrm{FePSe}_{3}$. The physical property and the phase diagram of $\mathrm{FePX} X_{3}$ under $\mathrm{HP}$ are discussed along with the structural analyses results.

\section{Results}

Pressure-induced large-volume collapse. $\mathrm{FePX}_{3}(X=\mathrm{S}, \mathrm{Se})$ compounds adopt a $2 \mathrm{D}$ crystal structure with $\mathrm{Fe}^{2+}$ ions arranged in a nearly perfect honeycomb lattice and weak interlayer van der Walls interactions ${ }^{28}$. Figure $1 \mathrm{a}, \mathrm{b}$ display the crystal structure of the low-pressure (LP) phase of $\mathrm{FePS}_{3}$ and $\mathrm{FePSe}_{3}$ with an ideal trigonal symmetry. Each layer is composed of two layers of S/Se atoms, octahedrally coordinated $\mathrm{Fe}^{2+}$ ions, and phosphorous pairs. All the $\mathrm{FeX}_{6}$ octahedra share their edges with three neighbors to form the honeycomb lattice, and with $\mathrm{P}=\mathrm{P}$ dimers locating in the center of the sixfold mesh. The shortest $\mathrm{Fe}-\mathrm{Fe}$ distances in $\mathrm{FePX}_{3}$ are $\sim 3.44 \AA$ for $X=\mathrm{S}$ and $3.62 \AA$ for $X=\mathrm{Se}$, respectively, and both compounds are narrow-band semiconductors at ambient conditions. Under compression, $\mathrm{FeP} X_{3}$ undergo isostructural or quasi-isostructural phase transitions at $\sim 13 \mathrm{GPa}$ for $X=\mathrm{S}$ and $\sim 8 \mathrm{GPa}$ for $X=$ Se, respectively (Supplementary Fig. 1). The discontinuous shifts of the diffraction peaks $\left((113) /(20-2)\right.$ of $\mathrm{FePS}_{3}$ and (113) of $\mathrm{FePSe}_{3}$, as shown in Fig. 1c, d) indicate abruptly shrinking of the lattice parameters concomitant with the pressure-driven phase transition, in particular, the collapses in the $a b$-plane lattice spacings.

Judging from the X-ray diffraction (XRD) patterns, monoclinic unit cells with the space group $C 2 / m$ were adopted for the Le Bail fitting analyses for both $\mathrm{FePS}_{3}$ and $\mathrm{FePSe}_{3}$ (Supplementary Fig. 2). Figure 1e presents the evolution of the cell volumes of $\mathrm{FePX}$ as a function of applied pressure. Large cell volume decreases $(10.5 \%$ for $X=\mathrm{S}$ and $10.6 \%$ for $X=\mathrm{Se}$ ) dominate the $P-V$ profiles along with the first-order phase transitions, indicating remarkable changes of the atomic arrangement. Such large-volume collapses $(>5 \%)$ are usually associated with the pressure-driven SCO on the origin ${ }^{21,22}$. The decrease of $\mathrm{Fe}^{2+}$ ionic radii from $0.78 \AA$ (high spin (HS), $S=2$ ) to $0.61 \AA$ (low spin (LS), $S=0$ ) results in the shortening of $\mathrm{Fe}-(\mathrm{S} / \mathrm{Se})$ bond lengths and the possible symmetry lowering of the hexagonal lattice. To sententiously analysis the anisotropic compressibility of $\mathrm{FePX}_{3}$, a pseudo-trigonal space group R-3 is adopted for the XRD refinements of $\mathrm{FePSe}_{3}$ (Fig. 1f). The dramatic decreases of $a$ and $b$ during the phase transition clearly indicate that the volume collapses origin from the shrinkages of the $\mathrm{Fe}^{2+}$ honeycomb lattices, which also indicate the formation of in-plane intermetallic Fe-Fe bonding. Similar inplane lattice collapse is also observed in $\mathrm{FePS}_{3}$ with monoclinic symmetry (Supplementary Fig. 3).

Pressure-induced spin-crossover. We have proposed that the pressure-driven cooperative SCO, i.e., large-volume collapse accompanied with SCO and IMT, should be a universal behavior of $3 d$ TM chalcogenides ${ }^{26,27}$. FePX $X_{3}$, as an ideal low-dimensional confined system similar to $\mathrm{MnPX}_{3}{ }^{27}$, thus is highly expected to achieve a nonmagnetic HP phase via pressure-driven cooperative SCO. In situ X-ray emission spectroscopy (XES) ${ }^{23}$ measurements were performed to study the spin-state transitions of $\mathrm{FePX} X_{3}$ under $\mathrm{HP}$ and the results are presented in Fig. 2a, b. It is generally recognized that the $\mathrm{K}_{\beta}$ lines are the characteristic emissions originated from the $3 p \rightarrow 1 s$ transition, and the shapes of $\mathrm{K}_{\beta}$ lines are determined by the interactions between the $3 p$ core hole and the 

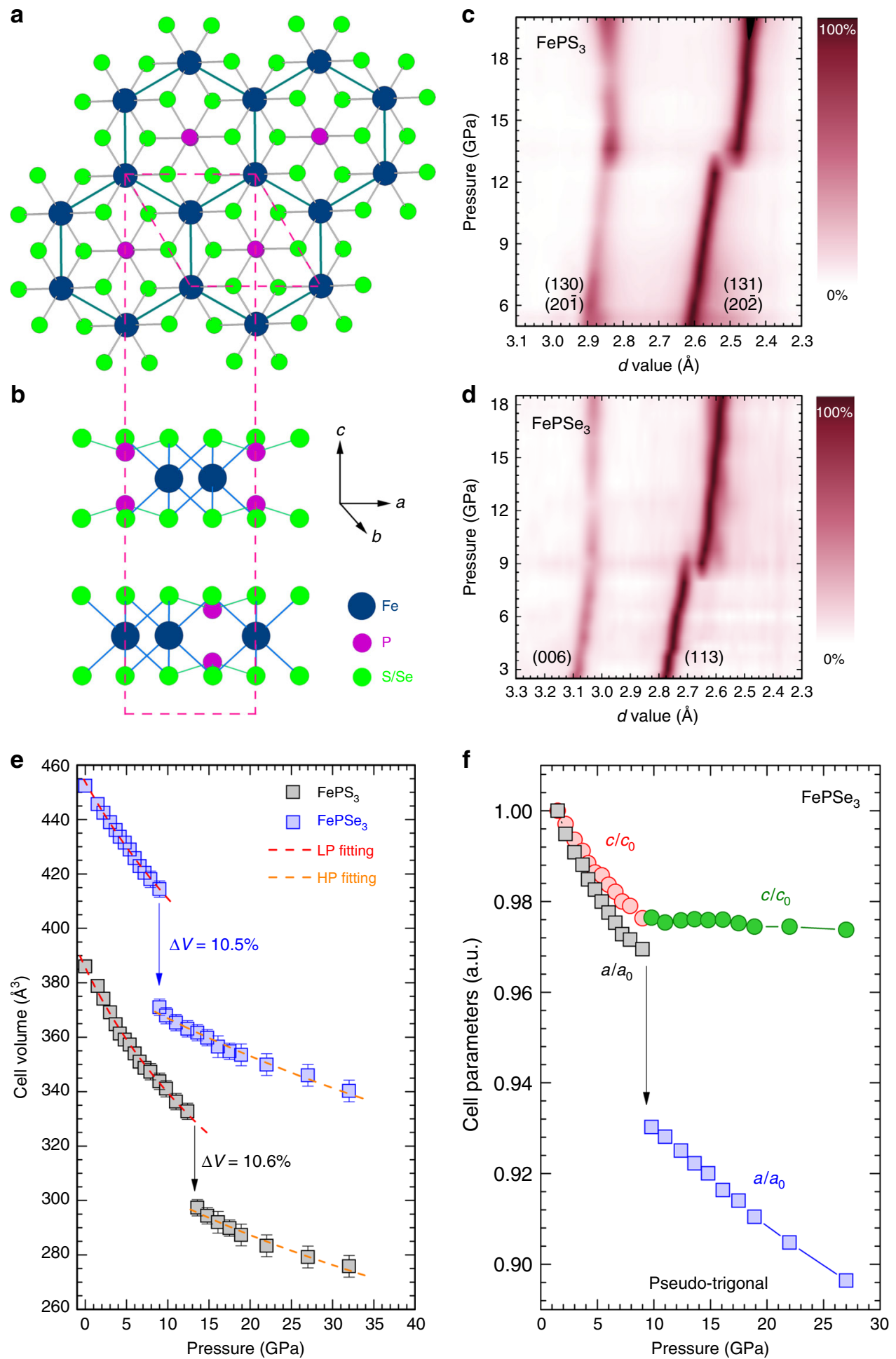

Fig. 1 The crystal structure and pressure-induced phase transition of FePX $X_{3}$ a, b Ambient crystal structure of FePX $(X=\mathrm{S}, \mathrm{Se})$ viewed along $c$ - and $a / b$ axis showing the layered structure feature and the $\mathrm{Fe}^{2+}$ honeycomb lattice. $\mathbf{c}, \mathbf{d}$ Two-dimensional PXRD data showing abrupt pressure-induced changes of the (131) and (20-2) peaks for $\mathrm{FePS}_{3}$ and the (113) peak for $\mathrm{FePSe}_{3}$, respectively. e The derived cell volume values as a function of applied pressure for the $\mathrm{LP}$ and HP phases of $\mathrm{FePS}_{3}$ and FePSe . The derived bulk moduli are: $B_{0}=61.1(2)$ and 162(9) GPa for $\mathrm{LP}$ and $\mathrm{HPFePS} ; B_{0}=82.8(7)$ and $201(8) \mathrm{GPa}$ for $\mathrm{LP}$ and $\mathrm{HP} \mathrm{FePSe}{ }_{3}$, respectively. Error bars represent \pm S.D. $\mathbf{f}$ The cell parameters of $\mathrm{FePSe}_{3}$ with a pseudo-trigonal unit cell showing an in-plane collapse along with the pressure-induced phase transition

partially filled $3 d$ shell electrons. Therefore, it allows qualitative distinction of the HS/LS states from the relative intensities of satellite $\mathrm{K}_{\beta}{ }^{\prime}$ and the peak positions of $\mathrm{K}_{\beta 1,3}$. In the LP phases of $\mathrm{FePX} X_{3}$, well-defined $\mathrm{K}_{\beta}{ }^{\prime}$ satellite peaks are observed with a HS state $(S=2)$. Concomitant with the structural phase transition, the $\mathrm{K}_{\beta}{ }^{\prime}$ peaks drop suddenly and the $\mathrm{K}_{\beta 1,3}$ lines shift to lower energy correspondingly, both of which indicate the occurrence of $\mathrm{SCO}$ of $\mathrm{Fe}^{2+}$ within the honeycomb lattice.

Quantitative analysis of the XES data has been conducted using the integrals of the absolute values of the difference spectra (IAD) 
$\operatorname{method}^{29}$, and the differential curves are provided in Supplementary Fig. 4. The pressure dependence of the $\mathrm{Fe}^{2+}$ spin state in $\mathrm{FePX}_{3}$ is presented in Fig. 2c. At ambient conditions, the two compounds have a HS state of $\mathrm{Fe}^{2+}\left(3 d^{6}\right)$ with $S=2(\mathrm{IAD}=0)$. The HS state of $\mathrm{Fe}^{2+}$ is stable until the applied pressure exceeds the LP-to-HP structural transition points. The IAD values of $\mathrm{FePX}_{3}$ increase to $\sim 2.5(S=0)$ abruptly, indicating a complete collapse of the $\mathrm{Fe}^{2+}$ spin moments. Like the pressure effect on $\mathrm{MnPX}_{3}$ system $^{27}$, the abrupt pressure-driven SCO in $\mathrm{FePX}$ is attributed to their $2 \mathrm{D}$ crystal structures, where the spins located on the honeycomb lattice can more collectively communicate with neighboring spins than those on $3 \mathrm{D}$ lattices.

Pressure-induced semiconductor-to-metal transition. In situ transport measurements of $\mathrm{FeP} X_{3}$ show IMT along with the pressure-driven structural phase transition and SCO (as shown in Fig. 3). During the IMT process, the electrical resistances of $\mathrm{FePX}_{3}$ drop by more than five orders of magnitude at room temperature. The $R-T$ curves under HP also indicate a change from a semi-conductive behavior of the LP phases to metallic behavior of the HP phases, concomitant with the structural and electronic configuration transitions. Since the Fe-Fe intermetallic bonding can only form in the honeycomb layer, it is reasonable to see that the HP phases exhibit a bad-metal and an anisotropic transporting behavior. The conductivity measurements on the single crystal of $\mathrm{FePSe}_{3}$ confirm that the in-plane metallization contributes most to the overall IMT phenomenon (Fig. 3c). What needs to be pointed out here is, all the data obtained in our transport measurements are original resistances rather than absolute resistivity, which may be converted to each other by normalizing the ambient resistivity values inside and outside the diamond anvil cell (DAC).

Observation of superconductivity. Figure $4 \mathrm{a}$ presents the resistance $R$ of $\mathrm{FePSe}_{3}$ as a function of temperature $T$ for pressure in the range of 9.0-29.6 GPa. A sharp drop of $R$ at an onset tem-
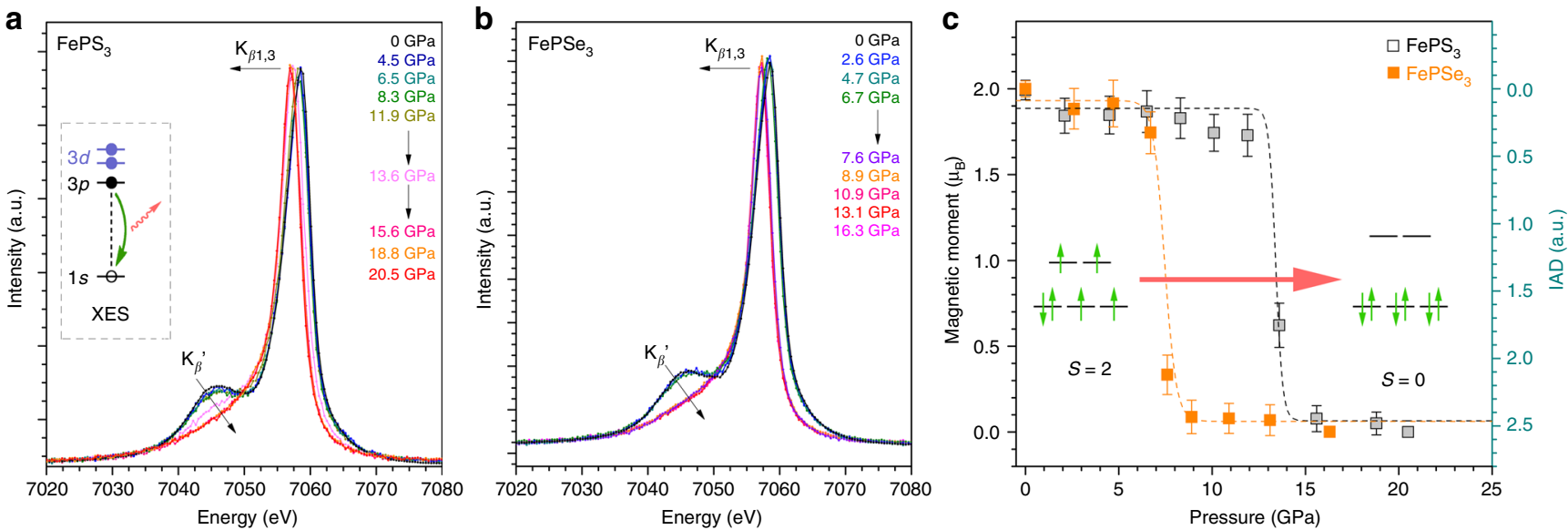

Fig. 2 XES data of $\mathrm{FePS}_{3}$ and $\mathrm{FePSe}_{3}$ showing the spin-crossover of Fe ${ }^{2+}$ under compression. $\mathbf{a}, \mathbf{b}$ Fe $\mathrm{K}_{\beta}$ XES of FePS $\mathrm{Fe}_{3}$ and $\mathrm{FePSe}_{3}$ as a function of applied pressure. Inset of a shows the XES process from $3 p$ to $1 s$ orbital, and the relationship with $3 d$ spin state. $\mathbf{c}$ The derived IAD values and corresponding magnetic moment numbers of $\mathrm{FePS}_{3}$ and $\mathrm{FePSe}_{3}$ as a function of pressure. Error bars represent \pm S.D. estimated for the IAD analyses

a

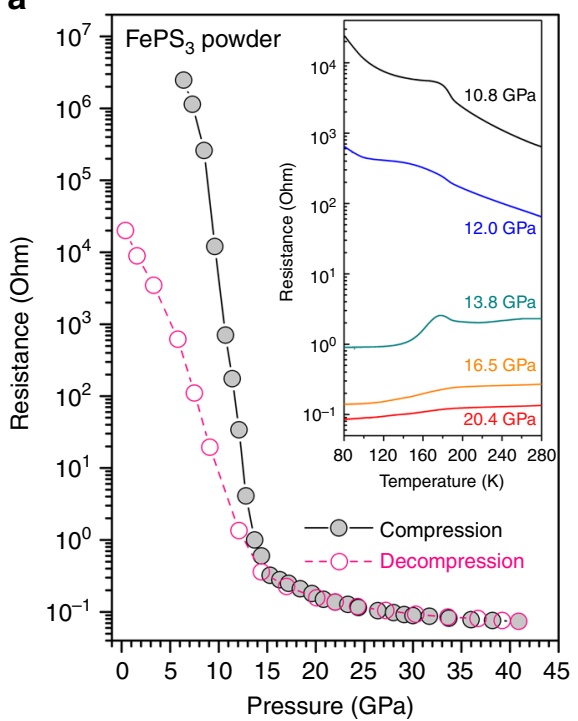

b

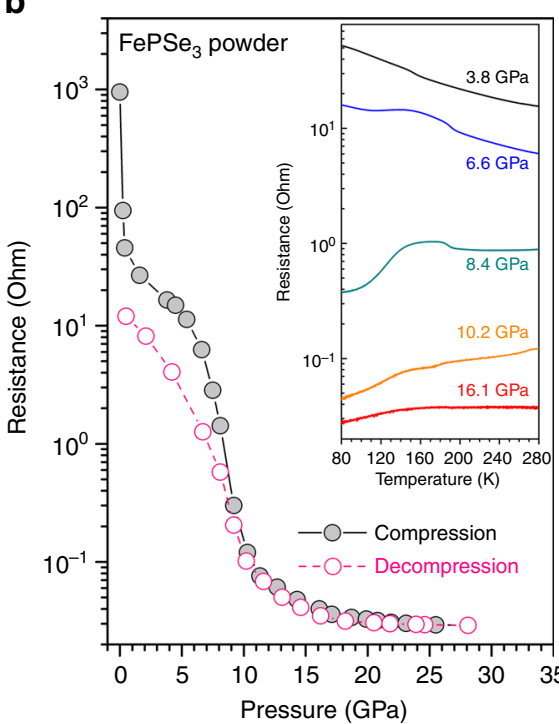

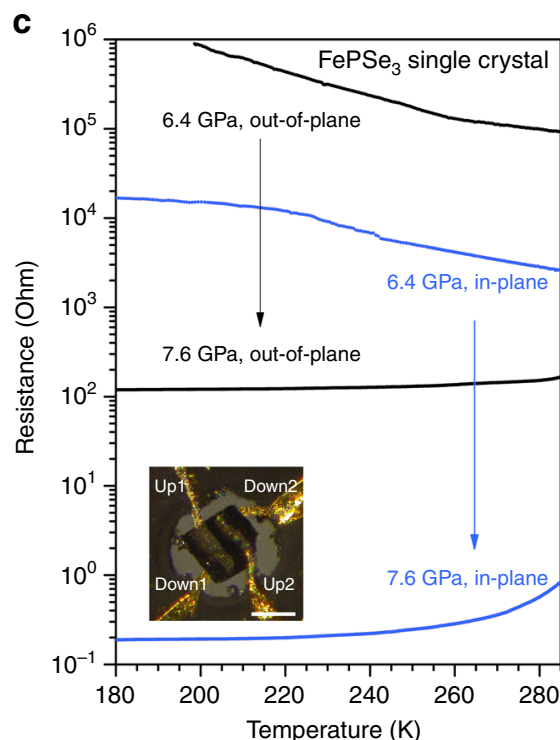

Fig. 3 Pressure-induced semiconductor-to-metal transitions in $\mathrm{FePS}_{3}$ and $\mathrm{FePSe}_{3}$. $\mathbf{a}, \mathbf{b}$ Electrical resistances of compressed powder of FePX $3(X=\mathrm{S}, \mathrm{Se})$ as a function of pressure. Insets of $\mathbf{a}$, $\mathbf{b}$ show the temperature dependence $(R-T)$ of the resistance of $\mathrm{FePS}_{3}$ and FePSe $\mathrm{F}_{3}$ under high pressure, respectively. $\mathbf{c}$ Electrical resistance measurements on a $\mathrm{FePSe}_{3}$ single crystal showing an in-plane metallization behavior. Inset of $\mathbf{c}$ shows the photograph of the FePSe $\mathrm{F}_{3}$ single crystal with four Au probes inside a DAC. The scale bar is $100 \mu \mathrm{m}$ 
perature of $\sim 2.5 \mathrm{~K}$ is clearly observed near the critical pressure $P_{\mathrm{c}} \approx 9.0 \mathrm{GPa}$, which is just above the pressure-driven structural phase transition point and indicates the occurrence of SC within the HP phase of $\mathrm{FePSe}_{3}$. During further pressure increase, the onset $T_{\mathrm{c}}$ suddenly increases to $5.5 \mathrm{~K}$ above $20 \mathrm{GPa}$, while the transition becomes much broader. The superconducting transition becomes sharper with zero resistivity reached at $3 \mathrm{~K}$ around $30 \mathrm{GPa}$. Above $29.6 \mathrm{GPa}$, the $T_{\mathrm{c}}$ of $\mathrm{FePSe}_{3}$ shows a slight tendency to decrease with the pressure increase, but the SC state remains up to $41.4 \mathrm{GPa}$ (the highest pressure in our measurements, as shown in Fig. $4 \mathrm{~b}$ ). The zero resistance has been reached in the experiment for most of the pressure points. All these evidences highlight the fact that the emerging SC state is an intrinsic behavior of the HP phase of $\mathrm{FePSe}_{3}$.

We have also applied magnetic field to suppress the SC. Under the constant pressure of $29.6 \mathrm{GPa}$, temperature dependences of resistance of $\mathrm{FePSe}_{3}$ at various magnetic fields are shown in Fig. 4c. During the increase of the magnetic field, the transition is gradually suppressed, which further confirms that it is the superconducting transition. We have obtained the upper critical field $\left(H_{\mathrm{c} 2}\right)$ as shown in the inset of Fig. 4c. The $H_{\mathrm{c} 2}$ shows an upturn curve, which may be related to the multiband SC in a $2 \mathrm{D}$ system. The $T_{\mathrm{c}}$ values obtained from the above measurements along with the structural and spin-state transitions are plotted in the temperature-pressure phase diagram of $\mathrm{FePSe}_{3}$, as shown in Fig. $4 \mathrm{~d}$. The application of HP drives the phase transition from the LP, HS, and magnetic insulator (or semiconductor) state to a HP, LS, nonmagnetic, and metallic state in $\mathrm{FePSe}_{3}$. SC emerges with a starting $T_{\mathrm{c}} \sim 2.5 \mathrm{~K}$ at $9.0 \mathrm{GPa}$ and a maximum $T_{\mathrm{c}} \sim 5.5 \mathrm{~K}$ at $29.6 \mathrm{GPa}$ and sustains within the HP phase of $\mathrm{FePSe}_{3}$ up to 40 GPa. The SC originated from the parent AFM materials has strong similarities with the unconventional superconductors such as high- $T_{\mathrm{c}}$ cuprate SCs, FeSCs, and heavy Fermion SCs. These findings demonstrate a successful exploration of new nonmagnetic phases with SC via pressure-driven spin-quenching. The positive Hall coefficient measured at $10 \mathrm{~K}$ indicates that holes are the main carriers in the SC phase. The sudden increase of $T_{\mathrm{c}}$ above $20 \mathrm{GPa}$, coincident with the anomaly of the Hall coefficient, indicates a possible Fermi surface reconstruction.

\section{Discussion}

The honeycomb layers of $\mathrm{FePX} X_{3}$ can survive through the pressure-driven quasi-isostructural phase transition. However, the in-plane lattice collapse is proved to be anisotropic and the symmetry breaking down to pseudo-trigonal monoclinic is expected to happen, as shown in Fig. 5a, b. First, the formation of shorter $\mathrm{Fe}-\mathrm{Fe}$ intermetallic bonding favors the occurrence of cooperative SCO and is consistent with the IMT scenario ${ }^{27}$. Second, the results of in situ-extended X-ray absorption fine structure measurements on Fe K-edge support the HP-mode-2, where the $\mathrm{Fe}-\mathrm{Fe}$ bonds split into two groups: an intermetallic bond with the $\mathrm{Fe}-\mathrm{Fe}$ distance of $3.24 \AA$ and an elongated $\mathrm{Fe}-\mathrm{Fe}$
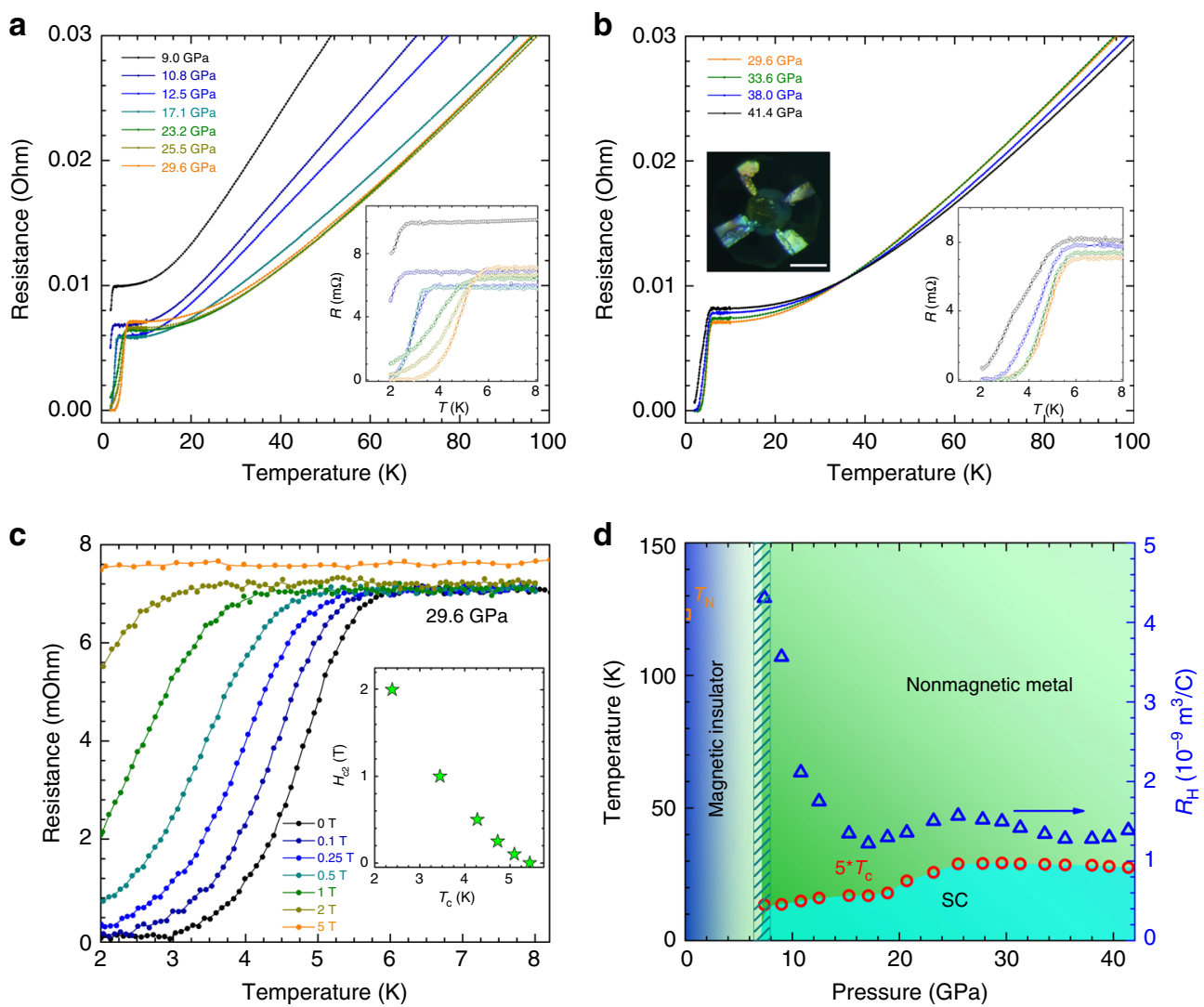

Fig. 4 Emergence of superconductivity under high pressure and temperature-pressure phase diagram in FePSe 3 . a The in-plane electrical resistivity of $\mathrm{FePSe}_{3}$ single crystal as a function of temperature, and applied pressures 9.0, 10.8, 12.5, 17.1, 23.2, 25.5, and 29.6 GPa. b The in-plane electrical resistivity of $\mathrm{FePSe}_{3}$ single crystal as a function of temperature, and applied pressures $29.6,33.6,38.0$, and $41.4 \mathrm{GPa}$. Insets of $\mathbf{a}$, $\mathbf{b}$ show the enlarged low-temperature parts of the measured resistivity. Line colors are the same as those used in $\mathbf{a}, \mathbf{b}$. Inset of $\mathbf{b}$ shows the photograph of a single-crystal FePSe $\mathrm{F}_{3}$ inside a DAC for resistivity measurements. The scale bar is $100 \mu \mathrm{m}$. c The temperature dependence of the electrical resistivity of $\mathrm{FePSe}_{3}$ at $29.6 \mathrm{GPa}$ under magnetic fields of $0,0.1,0.25,0.5,1,2$, and $5 \mathrm{~T}$. Inset shows the field dependence of $T_{\mathrm{c}}$ for $\mathrm{FePSe}_{3}$ at $29.6 \mathrm{GPa}$. $\mathbf{d} \mathrm{Temperature-pressure} \mathrm{phase} \mathrm{diagram} \mathrm{of} \mathrm{FePSe}_{3}$, where solid circles represent the pressure dependence of the onset superconducting transition temperatures $\left(5^{\star} T_{\mathrm{c}}\right.$ ), and the blue triangles represent the pressure dependence of Hall coefficient. $T_{\mathrm{N}}$ represents the Neel temperature of $\mathrm{FePSe}_{3}$ 


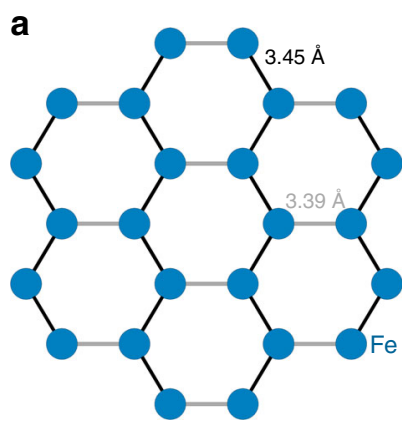

HP-mode-1

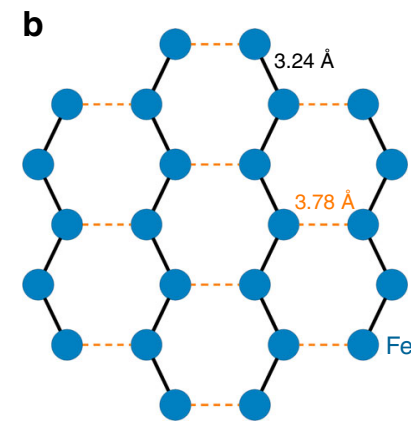

HP-mode-2

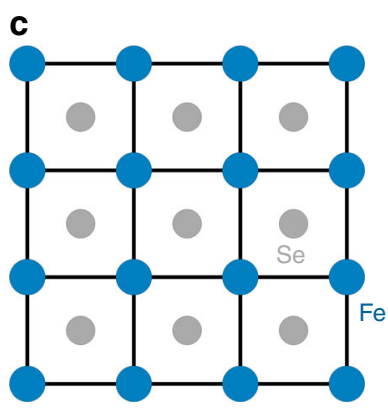

FeSe-type

Fig. 5 High-pressure structure modes of $\mathrm{Fe}^{2+}$ honeycomb lattice and comparison with FeSe-type tetragonal Fe $\mathrm{e}^{2+}$ lattice. a The proposed HP-mode- 1 for $\mathrm{FePSe}_{3}$ with slightly distorted $\mathrm{Fe}^{2+}$ honeycomb lattice. $\mathbf{b}$ The proposed HP-mode- 2 for FePSe 3 with seriously distorted Fe $\mathrm{Fe}^{2+}$ honeycomb lattice. The shortest or the near-shortest Fe-Fe bond lengths are highlighted by black and gray lines in the HP-mode-1 and black and yellow lines in the HP-mode-2, respectively. c Tetragonal $\mathrm{Fe}^{2+}$ lattice in most Fe-based high- $T_{\mathrm{c}}$ superconductors with FeSe-type layered structures

distance of $3.78 \AA$, as shown in Supplementary Fig. 5. Therefore, the HP phases of $\mathrm{FePX}_{3}$ adopt exactly a distorted honeycomb-like $\mathrm{Fe}^{2+}$ lattice. The valence of iron is verified to be +2 in the HP phase of $\mathrm{FePSe}_{3}$ (Supplementary Fig. 6), and the increases of the pre-edge peaks indicate the distortion of $\mathrm{FeX}_{6}$ octahedra along with the formation of $\mathrm{Fe}-\mathrm{Fe}$ intermetallic bonding. Figure $5 \mathrm{c}$ presents the typical tetragonal lattice of FeSe, which is adopted by all the high- $T_{\mathrm{c}}$ FeSCs discovered so far. The HP phase of $\mathrm{FePSe}_{3}$ with an $\mathrm{Fe}^{2+}$ honeycomb lattice presents a unique example of FeSCs without a FeSe-type tetragonal or tetragonal-like lattice. Obviously, the discovery of $3 d$ TM-based SC in a new structure prototype will greatly arouse the passion of pursuing high- $T_{c} S C s$.

We have made significant efforts to determine the HP crystal structures of $\mathrm{FePX}_{3}$, including repeating powder and singlecrystal XRD measurements. However, the detailed structural features, such as the exact space group (symmetry) and atomic positions, are still not well-determined currently due to the difficulty of handling the less than perfect-quality data obtained under HP conditions. The HP crystal structure requires more efforts to be precisely determined in future investigations. In our experiments, $\mathrm{SC}$ is only observed in $\mathrm{FePSe}_{3}$. The HP phase of $\mathrm{FePS}_{3}$ may be either non-superconducting or with a very low $T_{\mathrm{c}}$ beyond our measurement capability (below $2 \mathrm{~K}$ ). Moreover, the determination of a detailed $P-T$ phase diagram of $\mathrm{FeP} X_{3}$ involving the AFM transition temperatures is now in progress, which may provide more evidence to the SC mechanism within an $\mathrm{Fe}^{2+}$ honeycomb lattice and most importantly whether it is an unconventional SC or not. We would also point out that the SC, the route to $\mathrm{SC}$, and the unique $\mathrm{Fe}^{2+}$ lattice in $\mathrm{FePSe}_{3}$ are obviously different from those in superconducting, nonmagnetic iron metal under compression ${ }^{30}$. There are many other candidates with low-dimensional $\mathrm{Fe}^{2+}$ lattices or $3 d$ TM-based lowdimensional lattices with $d^{6}$ electronic configuration. In principle, they can be tuned to superconducting phases via pressure-driven SCO. Modern structure design and theoretical prediction could facilitate the exploration of such new high- $T_{c}$ SCs based on this principle.

\footnotetext{
Methods

Material syntheses. All chemicals were of reagent-grade quality. They were purchased from commercial sources and used as received. $\mathrm{FePS}_{3}$ and $\mathrm{FePSe}_{3}$ powders were synthesized by heating stoichiometric $\mathrm{Fe}, \mathrm{P}$, and $\mathrm{S} / \mathrm{Se}$ powders at 700 ${ }^{\circ} \mathrm{C}$ in sealed quartz tubes for $24 \mathrm{~h}$. High-quality single crystals were grown by chemical vapor transport method in a two-zone furnace ${ }^{31}$. For both compounds, the temperatures of the two zones were $720-700^{\circ} \mathrm{C}$ with iodine as the transport medium. The growth experiments were carried out for a week, and the resulting single crystals were platelets of $\sim 0.01-0.1 \mathrm{~mm}$ in thickness and $\sim 0.5-1 \mathrm{~mm}^{2}$ in size with black appearance.
}

High-pressure structural characterizations. A symmetric DAC with a pair of $300 \mu \mathrm{m}$ diameter culet-sized diamond anvils was used for all the in situ measurements under HP. Typically, a steel gasket was pre-indented to $40 \mu \mathrm{m}$ in thickness and a $120 \mu \mathrm{m}$ diameter hole was laser-drilled to serve as the sample chamber. $\mathrm{FePS}_{3}$ and $\mathrm{FePSe}_{3}$ powders were pre-compressed into pellets before use. In all the experiments, silicone oil was used as the pressure-transmitting medium (PTM). Ruby balls were used as the pressure gauge and the pressures were calibrated according to the ruby fluorescence method ${ }^{32}$. The in situ XRD measurements were performed at 16 BM-D station of the High Pressure Collaborative Access Team (HPCAT) at Advanced Photon Source (APS) of Argonne National Laboratory (ANL). A focused monochromatic X-ray beam with $\sim 5 \mu \mathrm{m}$ in diameter (full width at half maximum) and $0.3100 \AA$ wavelength was used, and the diffraction patterns were recorded by using a MAR345 image plate.

High-pressure spectral studies. HP XES experiments were performed at the 16 ID-D station of HPCAT. Be gaskets were used to confine and pressurize the sample and silicone oil was used as the PTM. X-ray absorption spectrum (XAS) measurements at the Fe K-edge were performed at the 20 ID-C, APS, ANL. A pair of nano-diamond anvils was used to avoid the diffraction glitches from regular single-crystal diamonds. No PTM was used to ensure the uniformity of the thickness.

High-pressure transport measurements. Electrical resistances of $\mathrm{FePS}_{3}$ and $\mathrm{FePSe}_{3}$ powders were measured using a four-point-probe resistance measurement system consisting of a Keithley 6221 current source, a 2182A nanovoltmeter, and a 7001 voltage/current switch. Cubic boron nitride layers were inserted into the DAC between the steel gasket and diamond anvils to provide electrical insulation. Four gold wires were arranged to contact the surface of the sample in the chamber.

We conducted the HP electric transport measurements on a $\mathrm{FePSe}_{3}$ single crystal under pressure by using the miniature diamond anvil cell ${ }^{33}$. Diamond anvils with $300 \mu \mathrm{m}$ culet and $c$-BN gasket with sample chambers of diameter $140 \mu \mathrm{m}$ were used. $\mathrm{A} \mathrm{FePSe}_{3}$ single crystal was cut with the dimensions of $60 \times 60 \times 10 \mu^{3}$. Four Pt wires were adhered to the sample using the silver epoxy. Daphne oil 7373 was used as the PTM. Resistance and Hall coefficient were measured using the Quantum Design PPMS-9.

Data analyses. The $2 \mathrm{D}$ XRD patterns were integrated using the Dioptas program $^{34}$. Unit cell parameter refinements were performed with FULLPROF program $^{35}$. The quantitative analyses of the XES data were carried out through IAD (IAD method) ${ }^{29}$

Data availability. All data supporting the findings of this work are available from the corresponding author on request.

Received: 30 December 2017 Accepted: 19 April 2018

Published online: 15 May 2018

\section{References}

1. Kamihara, Y., Watanabe, T., Hirano, M. \& Hosono, H. Iron-based layered superconductor $\mathrm{La}\left[\mathrm{O}_{1-x} \mathrm{~F}_{x}\right] \mathrm{FeAs}(x=0.05-0.12)$ with $T_{\mathrm{c}}=26 \mathrm{~K}$. J. Am. Chem. Soc. 130, 3296-3297 (2008). 
2. Ren, Z.-A. et al. Superconductivity at $55 \mathrm{~K}$ in iron-based F-doped layered quaternary compound $\mathrm{Sm}\left[\mathrm{O}_{1-x} \mathrm{~F}_{x}\right]$ FeAs. Chin. Phys. Lett. 25, 2215-2216 (2008).

3. Paglione, J. \& Greene, R. L. High-temperature superconductivity in iron-based materials. Nat. Phys. 6, 645-658 (2010).

4. Kotegawa, H., Sugawara, H. \& Tou, H. Abrupt emergence of pressure-induced superconductivity of $34 \mathrm{~K}$ in $\mathrm{SrFe}_{2} \mathrm{As}_{2}$ : a resistivity study under pressure. $J$. Phys. Soc. Jpn. 78, 013709 (2009)

5. Sun, Y.-L. et al. $\mathrm{Ba}_{2} \mathrm{Ti}_{2} \mathrm{Fe}_{2} \mathrm{As}_{4} \mathrm{O}$ : a new superconductor containing $\mathrm{Fe}_{2} \mathrm{As}_{2}$ layers and $\mathrm{Ti}_{2} \mathrm{O}$ sheets. J. Am. Chem. Soc. 134, 12893-12896 (2012).

6. Iyo, A. et al. New-structure-type Fe-based superconductors: $\mathrm{CaAFe}_{4} \mathrm{As}_{4}(A=\mathrm{K}$, $\mathrm{Rb}, \mathrm{Cs}$ ) and $\mathrm{Sr}_{A \mathrm{Fe}_{4}} \mathrm{As}_{4}$ ( $A=\mathrm{Rb}, \mathrm{Cs}$ ). J. Am. Chem. Soc. 138, 3410-3415 (2016).

7. Wang, Z.-C. et al. Superconductivity in $\mathrm{KCa}_{2} \mathrm{Fe}_{4} \mathrm{As}_{4} \mathrm{~F}_{2}$ with separate double $\mathrm{Fe}_{2} \mathrm{As}_{2}$ layers. J. Am. Chem. Soc. 138, 7856-7859 (2016).

8. Wang, C. et al. A new ZrCuSiAs-type superconductor: ThFeAsN. J. Am. Chem. Soc. 138, 2170-2173 (2016).

9. Ying, T. P. et al. Observation of superconductivity at $30 \sim 46 \mathrm{~K}$ in $\mathrm{A}_{x} \mathrm{Fe}_{2} \mathrm{Se}_{2}$ $(\mathrm{A}=\mathrm{Li}, \mathrm{Na}, \mathrm{Ba}, \mathrm{Sr}, \mathrm{Ca}, \mathrm{Yb}$, and Eu). Sci. Rep. 2, 426 (2012).

10. Ying, T. et al. Superconducting phases in potassium-intercalated iron selenides. J. Am. Chem. Soc. 135, 2951-2954 (2013).

11. Burrard-Lucas, M. et al. Enhancement of the superconducting transition temperature of FeSe by intercalation of a molecular spacer layer. Nat. Mater. 12, 15-19 (2013).

12. Lu, X. F. et al. Coexistence of superconductivity and antiferromagnetism in $\left(\mathrm{Li}_{0.8} \mathrm{Fe}_{0.2}\right)$ OHFeSe. Nat. Mater. 14, 325-329 (2015).

13. Drozdov, A. P., Erements, M. I., Troyan, I. A., Ksenofontov, V. \& Shylin, S. I. Conventional superconductivity at 203 kelvin at high pressures in the sulfur hydride system. Nature 525, 73-76 (2015).

14. Drozdov, A. P., Eremets, M. I. \& Troyan, I. A. Superconductivity above $100 \mathrm{~K}$ in $\mathrm{PH}_{3}$ at high pressures. Preprint at https://arxiv.org/abs/1508.06224 (2015).

15. Cheng, J.-G. et al. Pressure induced superconductivity on the border of magnetic order in MnP. Phys. Rev. Lett. 114, 117001 (2015).

16. Sun, L. et al. Re-emerging superconductivity at 48 kelvin in iron chalcogenides. Nature 483, 67-69 (2012)

17. Takahashi, H. et al. Superconductivity at $43 \mathrm{~K}$ in an iron-based layered compound $\mathrm{LaO}_{1-x} \mathrm{~F}_{x} \mathrm{FeAs}$. Nature 453, 376-378 (2008).

18. Chu, C. W. et al. Superconductivity above $150 \mathrm{~K}$ in $\mathrm{HgBa}_{2} \mathrm{Ca}_{2} \mathrm{Cu}_{3} \mathrm{O}_{8+\delta}$ at high pressures. Nature 365, 323-325 (1993).

19. Medvedev, S. et al. Electronic and magnetic phase diagram of $\beta-\mathrm{Fe}_{1.01} \mathrm{Se}$ with superconductivity at $36.7 \mathrm{~K}$ under pressure. Nat. Mater. 8, 630-633 (2009).

20. Rosner, H. et al. Magneto-elastic lattice collapse in $\mathrm{YCo}_{5}$. Nat. Phys. 2, 469-472 (2006).

21. Kuneš, J. et al. Collapse of magnetic moment drives the Mott transition in MnO. Nat. Mater. 7, 198-202 (2008).

22. Kimber, S. A. J. et al. Giant pressure-induced volume collapse in the pyrite mineral $\mathrm{MnS}_{2}$. Proc. Natl Acad. Sci. USA 111, 5106-5110 (2014).

23. Rueff, J.-P. et al. Pressure-induced high-spin to low-spin transition in FeS evidenced by X-ray emission spectroscopy. Phys. Rev. Lett. 82, 3284-3287 (1999).

24. Kawakami, T. et al. Spin transition in a four-coordinate iron oxide. Nat. Chem. 1, 371-376 (2009).

25. Cheng, J. et al. Charge disproportionation and the pressure-induced insulatormetal transition in cubic perovskite $\mathrm{PbCrO}_{3}$. Proc. Natl Acad. Sci. USA 112, 1670-1674 (2015)

26. Wang, Y. et al. Giant pressure-driven lattice collapse coupled with intermetallic bonding and spin-state transition in manganese chalcogenides. Angew. Chem. Int. Ed. Engl. 55, 10350-10353 (2016).

27. Wang, Y. et al. Pressure-driven cooperative spin-crossover, large-volume collapse, and semiconductor-to-metal transition in manganese(II) honeycomb lattices. J. Am. Chem. Soc. 138, 15751-15757 (2016).

28. Wiedenmann, A., Rossat-Mignod, J., Louisy, A., Brec, R. \& Rouxel, J. Neutron diffraction study of the layered compounds $\mathrm{MnPSe}_{3}$ and $\mathrm{FePSe}_{3}$. Solid State Commun. 40, 1067-1072 (1981).

29. Vankó, G. et al. Probing the $3 \mathrm{~d}$ spin momentum with X-ray emission spectroscopy: the case of molecular-spin transitions. J. Phys. Chem. B 110, 11647-11653 (2006).
30. Shimizu, K. et al. Superconductivity in the nonmagnetic state of iron under pressure. Nature 412, 316-318 (2001).

31. Klingen, V. W., Ott, R. \& Hahn, H. Preparation and properties of hexaghioand hexaselenohypodiphosphates. Z. Anorg. Allg. Chem. 396, 271-278 (1973).

32. Mao, H. K., Xu, J. \& Bell, P. M. Calibration of the ruby pressure gauge to 800 kbar under quasi-hydrostatic conditions. J. Geophys. Res. 91, 4673-4676 (1986).

33. Gavriliuk, A. G., Mironovich, A. A. \& Struzhkin, V. V. Miniature diamond anvil cell for broad range of high pressure measurements. Rev. Sci. Instrum. 80, 043906 (2009)

34. Prescher, C. \& Prakapenka, V. B. DIOPTAS: a program for reduction of twodimensional X-ray diffraction data and data exploration. High Press. Res. 35, 223-230 (2015).

35. Rodriguez-Carvajal, J. Recent advances in magnetic structure determination by neutron powder diffraction. Phys. B 192, 55-69 (1993).

\section{Acknowledgements}

HPCAT operations are supported by DOE-NNSA under award no. DE-NA0001974, with partial instrumentation funding by NSF. J. Ying, Y. Xiao and P. Chow acknowledge the support of DOE-BES/DMSE under Award DE-FG02-99ER45775. APS is supported by DOE-BES, under contract no. DE-AC02-06CH11357. The HP XAS measurements were performed at 20 ID-C, APS, ANL. This work is supported by National Natural Science Foundation of China (51527801 and U1530402). We gratefully acknowledge Professor T. Irifune for providing nanodiamonds for the HP XAS measurements, L.X. Yang (HPSTAR), C.J. Sun (XSD), and C. Park (HPCAT) for their support in the in situ HP measurements, and P. Cifligu (UNLV) for his help with the manuscript.

\section{Author contributions}

J.S., W.Y., V.V.S., and Y.Z. conceived the project. Y.W., J.Y., and Z.Z. designed the experiments. Z.Z. synthesized the powder and single-crystal samples. Y.W. performed the in situ XRD, XES, XAS, and resistance measurements. J.Y. carried out the SC and Hall coefficient measurements. N.L. and Q.Z. performed quantitative analyses of the XRD and XES data. Y.W., Z.Z., and J.Y. wrote and revised the paper. All authors participated in discussing the results and editing the manuscript.

\section{Additional information}

Supplementary Information accompanies this paper at https://doi.org/10.1038/s41467 018-04326-1.

Competing interests: The authors declare no competing interests.

Reprints and permission information is available online at http://npg.nature.com/ reprintsandpermissions/

Publisher's note: Springer Nature remains neutral with regard to jurisdictional claims in published maps and institutional affiliations.

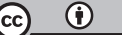

Open Access This article is licensed under a Creative Commons Attribution 4.0 International License, which permits use, sharing, adaptation, distribution and reproduction in any medium or format, as long as you give appropriate credit to the original author(s) and the source, provide a link to the Creative Commons license, and indicate if changes were made. The images or other third party material in this article are included in the article's Creative Commons license, unless indicated otherwise in a credit line to the material. If material is not included in the article's Creative Commons license and your intended use is not permitted by statutory regulation or exceeds the permitted use, you will need to obtain permission directly from the copyright holder. To view a copy of this license, visit http://creativecommons.org/ licenses/by/4.0/.

(C) The Author(s) 2018 